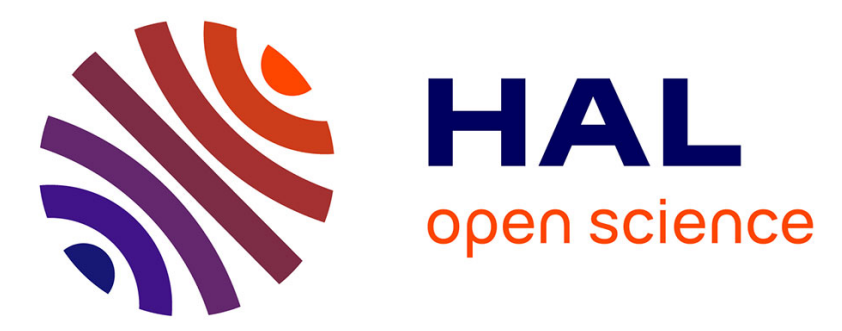

\title{
Measuring the off-axis angle and the rotational movements of phonating sperm whales using a single hydrophone
}

Christophe Laplanche, Olivier Adam, Maciej Lopatka, Jean-François Motsch

\section{- To cite this version:}

Christophe Laplanche, Olivier Adam, Maciej Lopatka, Jean-François Motsch. Measuring the off-axis angle and the rotational movements of phonating sperm whales using a single hydrophone. Journal of the Acoustical Society of America, 2006, Vol. 119, pp. 4074-4082. 10.1121/1.2184987 . hal-00797701

\section{HAL Id: hal-00797701 \\ https://hal.science/hal-00797701}

Submitted on 7 Mar 2013

HAL is a multi-disciplinary open access archive for the deposit and dissemination of scientific research documents, whether they are published or not. The documents may come from teaching and research institutions in France or abroad, or from public or private research centers.
L'archive ouverte pluridisciplinaire HAL, est destinée au dépôt et à la diffusion de documents scientifiques de niveau recherche, publiés ou non, émanant des établissements d'enseignement et de recherche français ou étrangers, des laboratoires publics ou privés. 


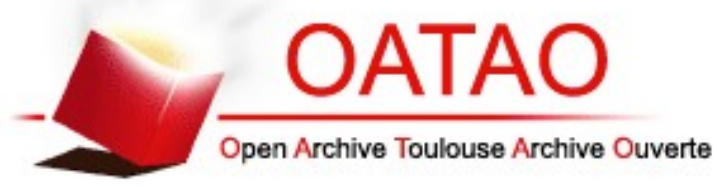

\section{Open Archive Toulouse Archive Ouverte (OATAO)}

OATAO is an open ac cess re pository that collects the work of Toulouse re searchers and makes it freely available over the web where possible.

This is an author-deposited ver sion p ublished in: http://oatao.univ-toulouse.fr/ Eprints ID: 5606

To link to this article: DOI: $10.1121 / 1.2184987$

http://dx.doi.org/10.1121/1.2184987

To cite this version: Laplanche, Christophe and Adam, Olivier and Lopatka, Maciej and Motsch, Jean -François Measuring the off-axis angle and the rotational movements of phonating sperm whales using a single hydrophone. (2006) The Journal of the Acoustical Society of America (JASA), Vol. $119\left(\mathrm{n}^{\circ} 6\right)$. pp. 4074-4082. ISSN 0001-4966

Any correspondence concerning this service should be sent to the repository administrator: staff-oatao@inp-toulouse.fr 


\title{
Measuring the off-axis angle and the rotational movements of phonating sperm whales using a single hydrophone
}

\author{
Christophe Laplanche, ${ }^{\text {a) }}$ Olivier Adam, Maciej Lopatka, and Jean-François Motsch \\ Laboratoire Images, Signaux et Systèmes Intelligents, Groupe Ingénierie des Signaux Neuro-Sensoriels, \\ Université Paris XII, France
}

\begin{abstract}
The common use of the bent-horn model of the sperm whale sound generator describes sperm whale clicks as the pulse series $\{p 0, p 1, p 2, p 3, \ldots\}$. Clicks, however, deviate from this standard when recorded using off-axis hydrophones. The existence of additional pulses within the $\{p 0, p 1, p 2, p 3, \ldots\}$ series can be explained still using the bent-horn model. Multiple reflections on the whale's frontal and distal sacs of the $p 0$ pulse lead to additional sets of pulses detectable using a farfield, off-axis hydrophone. The travel times of some of these additional pulses depend on the whale's orientation. The authors propose a method to estimate the off-axis angle of sperm whale clicks. They also propose a method to determine the nature of the movement (if it is pitch, yaw, or roll) of phonating sperm whales. The application of both methods requires the measurement of the travel time differences between pulses composing a sperm whale click. They lead, using a simple apparatus consisting of a single hydrophone at an unknown depth, to new measurements of the underwater movements of sperm whales. Using these methods shows that sperm whales would methodically scan seawater while searching for prey, by making periodic pitch and yaw movements in sync with their acoustic activity.
\end{abstract}

[DOI: 10.1121/1.2184987]

\section{INTRODUCTION}

Sperm whales (Physeter macrocephalus) mostly feed on meso- and bathypelagic squid and fish of various sizes and physical constitution (Kawakami, 1980; Whitehead, 2003). A 15-ton adult sperm whale would need to catch around half a ton of pelagic food in a day, assuming a daily consumption of $3 \%$ of its body weight (Lockyer, 1981). Sperm whales spend most of their time underwater and undertake long, deep dives to catch such an amount of prey (Miller et al., 2004; Watkins et al., 1999). However, the low-light, highpressure conditions reigning at these great depths make difficult the visual observation of foraging sperm whales. Consequently, one does not know precisely the technique(s) sperm whales would use to successfully seek and catch their food.

Nevertheless, scientists have developed many methods of studying the underwater behavior of deep-diving marine mammals (e.g., sperm whales), notably electronic tagging and passive acoustics. By using such methods, scientists have been collecting over the last decades many clues regarding the foraging behavior of sperm whales.

Sperm whales have a sound generator located in their snout (Madsen et al., 2002). The snout represents one quarter to one third the length of the whale (Clarke, 1978b; Nishiwaki et al., 1963), and the whale uses it primarily for biosonar purposes (Jaquet et al., 2001); Møhl et al., 2000; Whitehead and Weilgart, 1990). Sperm whales are indeed the most acoustically active toothed whales. They emit a series of transient sounds (clicks) when undertaking foraging dives

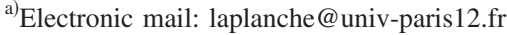

(Backus and Schevill, 1966). Such clicks would be of a duration, frequency content, source level, and directionality suited to echolocate their prey (Møhl et al., 2003, 2000).

Sperm whale dives are composed of a vertical descent, a hunt in a prey layer at depth, and a reascent (Miller et al., 2004). Sperm whales emit a long series of clicks at a slow rate (usual clicks, $0.45 \leqslant \mathrm{ICI}<2 \mathrm{~s}$, ICI denoting the interclick interval) during the descent. At depth, sperm whales emit usual clicks followed by clicks of increasing rate (creaks, ICI <0.45 s) and/or silences (Mullins et al., 1988; Zimmer et al., 2005b). It has been advanced that sperm whales emit usual clicks when searching for prey (denoted searching phase) and creaks when closing in on prey (denoted approach and terminal phases). They repeat this searching/approach/terminal pattern at depth (Laplanche et al., 2005; Madsen et al., 2005). According to Miller et al. (2004), sperm whales would keep crossing the prey layer during the searching phase and actively swim during the approach/terminal phases.

The sperm whale movements and precise use of their biosonar during these searching/approach/terminal phases are not perfectly understood yet. Laplanche et al. (2005) have proposed a model describing the sperm whale vertical movements and biosonar use during the searching phase. This model is, however, only partially correct and the authors offer some clarifications below. They propose a passive acoustic technique, requiring a single hydrophone, to determine the nature of the movements of diving sperm whales (i.e., if it is a pitch, yaw, or roll movement). They also present a new technique to estimate the off-axis angle of sperm whale clicks. The application of both techniques is grounded on a close description of the waveform of sperm 


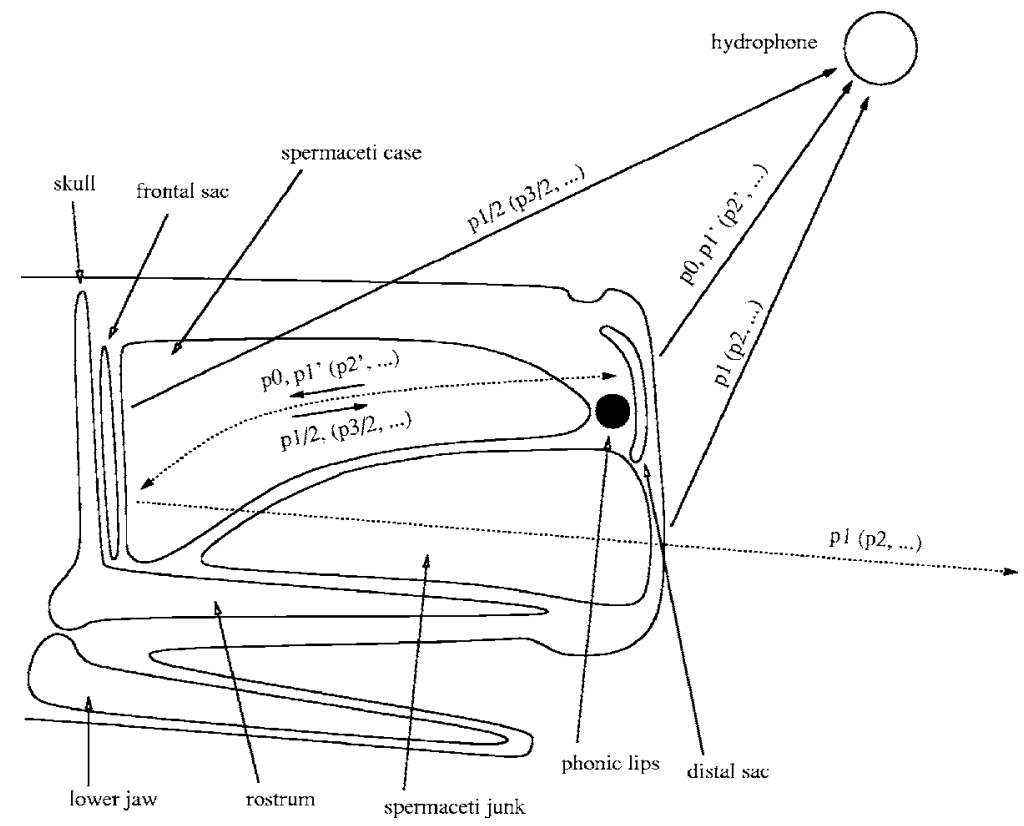

FIG. 1. The initial pulse $p 0$ is generated by the phonic lips, transmits through the spermaceti case, and reflects on the frontal sac into the $p 1 / 2$ pulse. The $p 1 / 2$ pulse transmits (1) through the junk and leads to the echolocation pulse $p 1,(2)$ back through the spermaceti case, reflects on the distal sac, and leads to the distal pulse $p 1^{\prime}$, and (3) through the whale's body to the receiver. The $p i^{\prime}$ pulses $(i \geqslant 1)$ then recursively lead to the $p(2 i$ $+1) / 2, p(i+1)$, and $p(i+1)^{\prime}$ pulses.

whale clicks, as predicted by the standard model of the sperm whale sound generator. The authors show that sperm whales synchronize their movements with their clicking activity. The authors then propose a more accurate model describing how sperm whales move and use their biosonar while searching for prey.

\section{METHODS}

\section{A. The leaky bent-horn model}

Today's hypothesis is that sperm whales generate their clicks with their snout (Norris and Harvey, 1972) according to the bent-horn model. This model, proposed by Møhl et al. (2003) and later confirmed by Zimmer et al. (2005b), successfully predicts the function and location of the various organs composing the sperm whale's snout. The sperm whale's snout is composed of the nasal circuitry (left and right nares), a connective organ (phonic lips), two air sacs (distal and frontal sacs), two spermaceti compartments (spermaceti case and junk), muscles, and blubber, as described by Clarke (1978b) and illustrated in Fig. 1. According to the bent-horn model, and in the notation of Møhl et al. (2003), clicks are generated by the phonic lips (leading to the $p 0$ pulse), roughly directed backward by the distal sac, focused by the spermaceti case, and reflected by the frontal sac. The frontal reflected pulse (labeled $p 1 / 2$ ) is channeled and projected forward by the spermaceti junk, leading to the outgoing $p 1$ pulse.

The use of this model also predicts that the sperm whale click waveform is multipulsed, i.e., composed of a series of pulses, $\{p 0, p 1, p 2, \ldots\}$ (Fig. 2, top). Indeed, the frontal reflection $p 1 / 2$ travels back in the spermaceti case and reflects on the distal sac (leading to a $p 1^{\prime}$ pulse). The distal reflection $p 1^{\prime}$ follows an acoustic path inside the snout of the whale similar to the one followed by $p 0$, leading to a frontal reflection $p 3 / 2$, the junk pulse $p 2$, and a distal reflection $p 2^{\prime}$, the distal reflection $p 2^{\prime}$ itself leading to the triplet $\left\{p 5 / 2, p 3, p 3^{\prime}\right\}$, and so on. The standard use of the bent-horn model predicts that the initial $p 0$ and the junk $\{p 1, p 2, p 3, \ldots\}$ pulses exit from the sperm whale's snout, explaining the observed multipulse structure of sperm whale clicks. The bent-horn model also successfully predicts the measured variations of the apparent source level of the $\{p 0, p 1\}$ pulses with the orientation of the hydrophone relative to the whale (Møhl et al., 2003; Zimmer et al., 2005b).

Using this model (or its Norris and Harvey ancestor), some researchers have described the $\{p 0, p 1, p 2, \ldots\}$ series as regular, the time of arrival difference between successive pulses $\{p i, p i+1\} \quad(i \geqslant 0)$ being a function of the whale's length (Goold, 1996; Gordon, 1991; Rhinelander and Dawson, 2004). However, the irregularity of the multipulse structure (i.e., variations of such time of arrival differences as well as the existence of additional pulses within the $\{p 0, p 1, p 2, \ldots\}$ frame) has been underlined by different re-
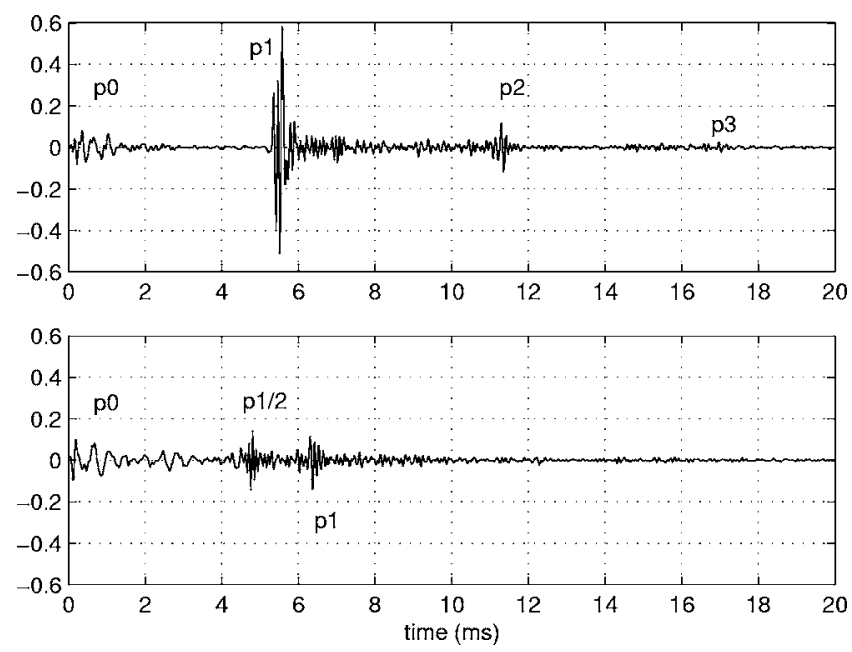

FIG. 2. Both clicks were emitted by the same sperm whale. Sperm whale clicks are often described as a series of regularly spaced pulses $\{p 0, p 1, p 2, \ldots\}$ (top). Clicks recorded in the farfield rarely fit to this model and are composed of a more complicate set of pulses $\{p 0, p 1 / 2, p 1, \ldots\}$ (bottom). 
searchers [see Bahl et al. (2002); Goold (1996); Møhl et al. (2003) for instance] without being elucidated.

The standard multipulse series $\{p 0, p 1, p 2, \ldots\}$ does not actually fully represent the waveform of sperm whale clicks. Clicks, when recorded off axis, deviate from this standard (Fig. 2, bottom). The authors propose a new and more complete description of the multipulse structure of sperm whale clicks, however, maintaining the standard bent-horn model, and describe what one could call the leaky bent-horn model. This description clarifies the discrepancies between the multipulse structure of clicks predicted by the standard model $(\{p 0, p 1, p 2, \ldots\})$ and what is actually observed in practice. Different researchers have reached similar conclusions (Zimmer et al., 2005a). The authors of the present work then do not reillustrate the soundness of the new model, as it has been convincingly done by Zimmer et al. (2005a). However, they briefly redefine it as a basis for the application described below. They also supplement the description of the sperm whale click multipulse structure proposed by Zimmer et al. (2005a), as it is not complete. For the sake of clarity, the authors of the present work use a notation similar to the notation that Zimmer et al. (2005a) introduced.

The standard use of the bent-horn model stipulates that only the initial and the junk pulses $\{p 0, p 1, p 2, p 3, \ldots\}$ exit from the sperm whale's snout. The leaky bent-horn model stipulates that (1) the above mentioned frontal $\{p 1 / 2, p 3 / 2, p 5 / 2, \ldots\}$ and distal $\left\{p 1^{\prime}, p 2^{\prime}, p 3^{\prime}, \ldots\right\}$ reflections also leak, and that (2) $\{p 0, p 1, p 2, p 3, \ldots\}$, $\{p 1 / 2, p 3 / 2, p 5 / 2, \ldots\}$, and $\left\{p 1^{\prime}, p 2^{\prime}, p 3^{\prime}, \ldots\right\}$ form distinct sets of pulses that can be separated using an off-axis hydrophone in the farfield. Zimmer et al. (2005a) demonstrated that the frontal $p 1 / 2$ pulse indeed leaks from the sperm whale's snout and that the $p 1$ pulse exits at the flat anterior surface of its junk. Zimmer et al. (2005a) also demonstrated that the travel time differences of the $p 1 / 2$ and $p 1$ pulses to the $p 0$ pulse depend on the orientation of the whale to the hydrophone. The authors of the present work do not reillustrate the soundness of such statements. They supplement the description of the waveform of a sperm whale click proposed by Zimmer et al. (2005a), asserting that it is also composed of the distal reflection $p 1^{\prime}$ and the additional sets of pulses $\{p 3 / 2, p 5 / 2, \ldots\},\{p 2, p 3 \ldots\}$, and $\left\{p 2^{\prime}, p 3^{\prime}, \ldots\right\}$, as described above. Such a description is discussed below.

\section{B. Definition of pulse delays and angles}

The delay of the $p y$ pulse on the $p x$ pulse is noted $\tau_{x y}$, simplified into $\tau_{y}$ if $p x=p 0$. The delays $\left\{\tau_{1^{\prime}}, \tau_{1^{\prime} 2^{\prime}}, \tau_{2^{\prime} 3^{\prime}}, \ldots\right\}$ are all equal to the travel time required by a pulse to travel twice between the distal and frontal sacs through the spermaceti case. Such a travel time is still denoted IPI by the authors, being the interpulse interval used in the literature regarding the sperm whale length estimation process mentioned above.

The delays $\left\{\tau_{1 / 2}, \tau_{3 / 2}, \ldots\right\}$ of the frontal pulses $\{p 1 / 2, p 3 / 2, \ldots\}$ to the initial $p 0$ pulse, and the delays $\left\{\tau_{1^{\prime} 1}, \tau_{2^{\prime} 2}, \ldots\right\}$ of the junk pulses $\{p 1, p 2, p 3, \ldots\}$ to the distal pulses $\left\{p 1^{\prime}, p 2^{\prime}, p 3^{\prime}, \ldots\right\}$ depend on the orientation of the whale to the hydrophone, as proven by Zimmer et al.

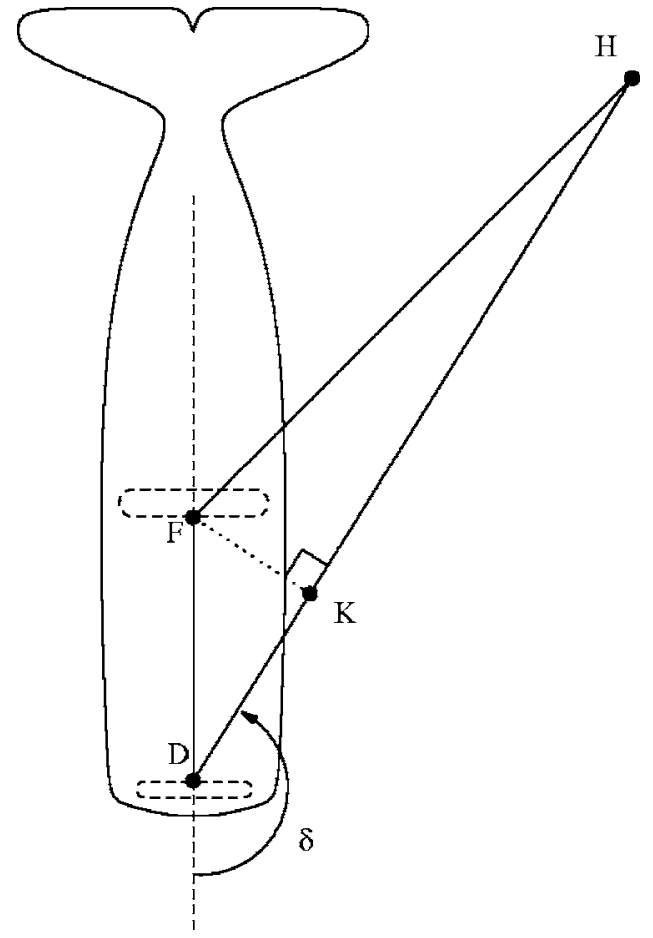

FIG. 3. The off-axis angle of the whale is defined as the angle between the whale dorsorostral axis and the line joining the whale to the hydrophone $H$. The point $D$ is at the center of the phonic lips (point of emission of the $p 0$ pulse). $F$ is the point of frontal reflection $p 0 \rightarrow p 1 / 2 . K$ is the orthogonal projection of $F$ on the line $(D H)$.

(2005a) regarding $\tau_{1 / 2}$ and $\tau_{1^{\prime} 1}$. Using a hydrophone at a fixed location in the farfield, $\left\{\tau_{1 / 2}, \tau_{3 / 2}, \ldots\right\}$ and $\left\{\tau_{1^{\prime} 1}, \tau_{2^{\prime} 2}, \ldots\right\}$, delays will change while the sperm whale moves underwater. Our aim in this work is to determine the nature of the whale's movements using the measurement of these delays. As described below, in the recordings used to illustrate this work, most of the time only pulses from the set $\left\{p 0, p 1 / 2, p 1, p 1^{\prime}\right\}$ are clearly detected. In the study of the whale's movements that follows, the authors have considered such pulses and used the delays $\left\{\tau_{1 / 2}\right.$, IPI, $\left.\tau_{1^{\prime} 1}\right\}$ only.

The authors consider in the following the reference frame of the whale. They define the pitch, yaw, and roll movements of the whale as rotations on its left-right, updown, and dorsorostral axes. As a difference, Zimmer et al. (2005b) and Laplanche et al. (2005) have both considered the sperm whale movements in the terrestrial reference frame. The off-axis angle of the whale (labeled $\delta \in[0, \pi]$, and estimated when the whale is emitting a click) is defined as the angle separating the whale dorso-rostral axis to the line joining the whale to the hydrophone (Fig. 3).

\section{Estimation of the off-axis angle of a click}

The off-axis angle of a whale represents its orientation to a reference point (e.g., the hydrophone). This angle varies with the whale's underwater movements, and, inversely, the variations of the off-axis angle of a whale provide information on the whale's underwater movements. The knowledge of this angle is critical in the following study, and the authors propose a simple method to carry out the estimation of the off-axis angle of a sperm whale click. 
The following demonstration applies for off-axis angles verifying $\delta \in[\pi / 2, \pi]$ (Fig. 3). The demonstration is slightly different if $\delta \in[0, \pi / 2]$, but leads to the same result, i.e., Eq. (5). The leaky bent-horn model predicts, as illustrated in Fig. 3 , that the $p 0$ pulse follows the path $[D K H]$, and the $p 1 / 2$ pulse follows the path $[D F H]$. The delay $\tau_{1 / 2}$ of the pulse $p 1 / 2$ on the pulse $p 0$ is then equal to $\left(\tau_{X Y}\right.$ denoting the travel time of a pulse on the path $[X Y]$ )

$$
\tau_{1 / 2}=\left[\tau_{D F}+\tau_{F H}\right]-\left[\tau_{D K}+\tau_{K H}\right] .
$$

The $p 0$ and $p 1 / 2$ pulses travel through the whale's body and through seawater to reach the hydrophone $H$. Refraction takes place during this transmission (Flewellen and Morris, 1978; Urick, 1983). Refraction in seawater leads to negligible variations of the quantity $\tau_{F H}-\tau_{K H}$, assuming a hydrophone in the farfield, the path $[K H]$ and $[F H]$ being close to each other. In this case,

$$
\tau_{F H}-\tau_{K H} \simeq 0 \text {. }
$$

In view of the previous statement IPI $=\tau_{1^{\prime}}$, then $\tau_{D F}=\mathrm{IPI} / 2$ leads to

$$
\tau_{1 / 2} \simeq \frac{\text { IPI }}{2}-\tau_{D K}
$$

The off-axis angle $\delta=(\overrightarrow{F D}, \overrightarrow{D K})$ can be found from $\cos \delta$ $\simeq-D K / F D$. One then assumes, given the dimension of the whale's body, that the curvature of the rays due to refraction in the whale's body lead to negligible variations on the delay $\tau_{D K}$. Then, assuming that the $p 0$ pulse travels along the path $[D K]$ in a straight line at the mean speed of sound $c_{\mathrm{sw}}=c_{D K}$, and along the path $[D F]$ at the mean speed of sound $c_{s}$, then

$$
\cos \delta \simeq-\frac{D K}{F D} \simeq-\frac{c_{\mathrm{sw}} \tau_{D K}}{c_{s} \mathrm{IPI} / 2} .
$$

Equations (3) and (4) lead to the following estimator of the off-axis angle of a click, as a function of the standard interpulse interval IPI and the delay $\tau_{1 / 2}$

$$
\delta \simeq \arccos \left(\frac{c_{\mathrm{sw}}}{c_{s}} \frac{2 \tau_{1 / 2}-\mathrm{IPI}}{\mathrm{IPI}}\right) .
$$

This formula also applies for $\delta \in[0, \pi / 2]$ ( $K$ being in that case the orthogonal projection of $D$ on the line $(F H)$ and choosing $c_{\mathrm{sw}}=c_{F K}$ as the mean speed of sound on the path $[F K])$. The mean speed of sound $c_{\mathrm{sw}}$ is a function of the off-axis angle, $c_{\mathrm{sw}}(\delta)$. The authors choose $c_{\mathrm{sw}}=c_{s}$ for onaxis angles $(\delta \in[0, \pi / 4]$ or $\delta \in[3 \pi / 4, \pi])$ and $c_{\mathrm{sw}}=c_{w}$ (naming $c_{w}$ the speed of sound in seawater) for off-axis angles $(\delta \in[\pi / 4,3 \pi / 4])$. Such an approximation is discussed below.

Equation (5) cannot be properly used to estimate the whale's off-axis angle if it is on-axis $\left(d \delta / d \tau_{1 / 2} \rightarrow+\infty\right.$ if $\tau_{1 / 2} \rightarrow 0$ or $\tau_{1 / 2} \rightarrow$ IPI). A click with a normalized delay [1 $\left.+\left(c_{s} / c_{w}\right) / \sqrt{2}\right] / 2 \leqslant \tau_{1 / 2} /$ IPI $\leqslant 1$ is on axis and the hydrophone is in front of the whale $(\delta \in[0, \pi / 4])$. A click with a normalized delay $0 \leqslant \tau_{1 / 2} / \mathrm{IPI} \leqslant\left[1-\left(c_{s} / c_{w}\right) / \sqrt{2}\right] / 2$ is on axis and the hydrophone is behind the whale $(\delta \in[3 \pi / 4, \pi])$. A click

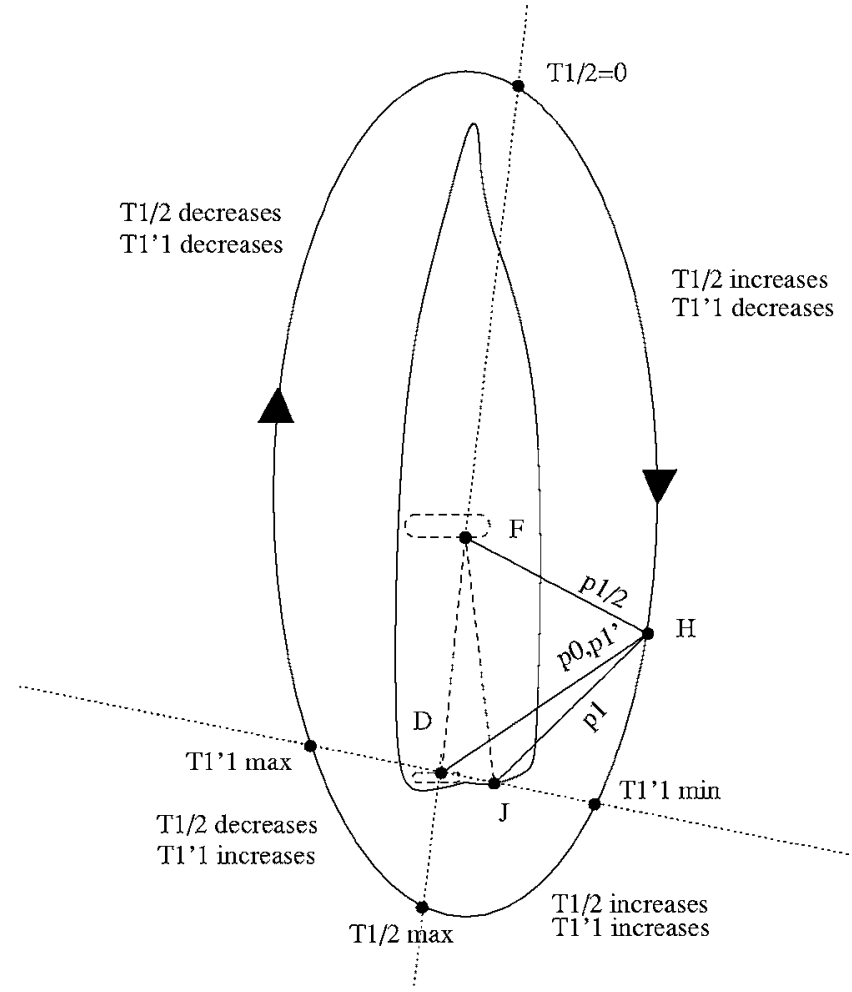

FIG. 4. $\tau_{1 / 2} \leqslant \mathrm{IPI} / 2$ if the hydrophone is behind the whale, $\tau_{1 / 2} \geqslant \mathrm{IPI} / 2$ if it is in front of the whale. $\tau_{1^{\prime} 1} \leqslant 0$ if the hydrophone is below the whale, $\tau_{1^{\prime} 1} \geqslant 0$ if it is above the whale. The sign of the variations of $\tau_{1 / 2}$ and $\tau_{1^{\prime} 1}$ during a pitch movement depend on the location of the hydrophone to the whale.

with a normalized delay $\left[1-\left(c_{s} / c_{w}\right) / \sqrt{2}\right] / 2 \leqslant \tau_{1 / 2} /$ IPI $<[1$ $\left.+\left(c_{s} / c_{w}\right) / \sqrt{2}\right] / 2$ is off axis $(\delta \in[\pi / 4,3 \pi / 4])$, and its offaxis angle can be estimated using

$$
\delta \simeq \arccos \left(\frac{c_{w}}{c_{s}} \frac{2 \tau_{1 / 2}-\text { IPI }}{\text { IPI }}\right) .
$$

\section{Travel time difference between $p 1$ and $p 1^{\prime}$}

The leaky bent-horn model also predicts, as illustrated in Fig. 4, that the $p 1$ pulse follows the path $[D F J H]$ and the $p 1^{\prime}$ pulse follows the path $[D F D H]$ to reach the hydrophone $H$. The algebraic delay $\tau_{1^{\prime} 1}$ of the echolocation pulse $p 1$ on the distal pulse $p 1^{\prime}$ also depends on the orientation of the whale to the hydrophone. As illustrated in Fig. 4 and demonstrated by Zimmer et al. (2005a),

$$
\tau_{1^{\prime} 1} \in\left[-D J / c_{w}, D J / c_{w}\right],
$$

where $J$ is at the center of the flat anterior surface of the junk. By writing the length $D J$ and the length $L_{\text {so }}$ of the spermaceti organ as functions of the whale's full length (noted $L$ ), $D J=\alpha(L) L$, and $L_{\mathrm{so}}=\beta(L) L$, approximating the length of the spermaceti organ as the length $D F$, and using $D F=c_{s} \mathrm{IPI} / 2$, then

$$
\frac{D J}{c_{w}}=\frac{\alpha(L)}{\beta(L)} \frac{c_{s}}{c_{w}} \frac{\text { IPI }}{2} .
$$

The authors then assume that the height to length ratio of the whale's spermaceti organ is equal to the height $\left(L_{h s}\right)$ to 

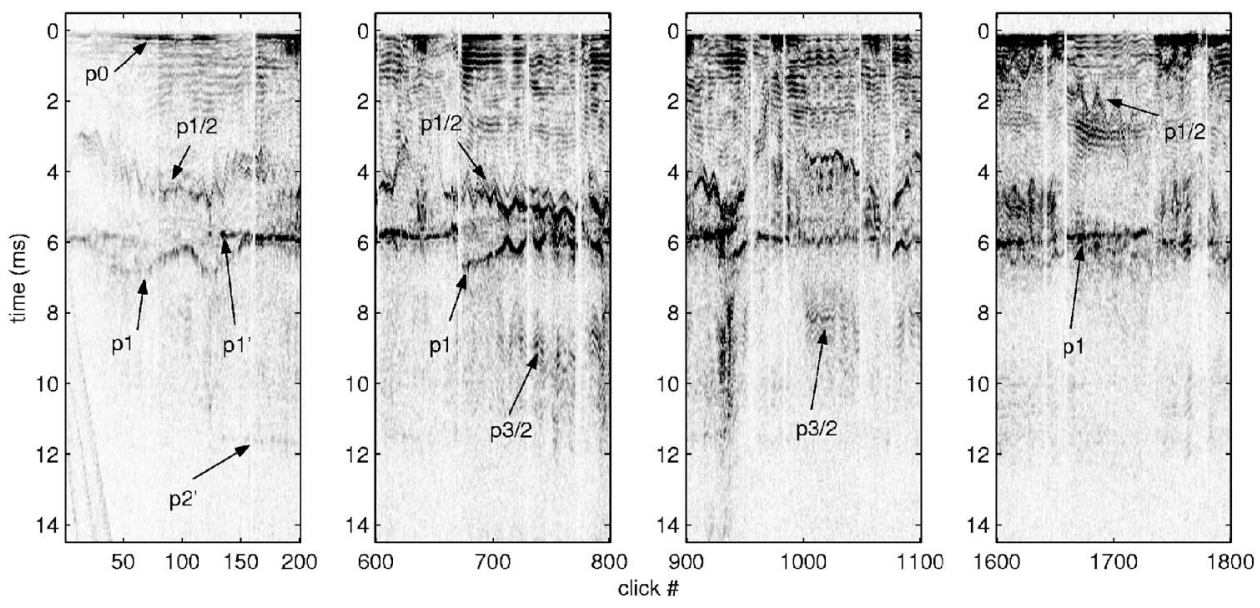

FIG. 5. The normalized, apparent level of $4 \times 200$ usual clicks emitted by a sperm whale during a single dive. Sea surface reflections are here visible from clicks $\# 0 \rightarrow \# 40$. A sperm whale click is composed of distal $\left\{p 0, p 1^{\prime}, p 2^{\prime}, \ldots\right\}, \quad$ frontal $\{p 1 / 2$, $p 3 / 2, \ldots\}$, and junk $\{p 1, p 2, \ldots\}$ pulses.

length $\left(L_{l s}\right)$ ratio of the whale's skull, such a hypothesis being discussed below. Using empirical relationships providing the height and length of the skull as functions of the whale's full length, $L_{h s}=\gamma(L) L$ and $L_{l s}=\zeta(L) L$, they find $D J / L_{\mathrm{so}}$ $=\alpha(L) / \beta(L)=\gamma(L) / \zeta(L)$. Linear regressions of the results provided for large whales $(11 \leqslant L \leqslant 17 \mathrm{~m})$ by Nishiwaki et al. (1963) lead to $\gamma(L) \simeq[12-L / 15] / 100$ and $\zeta(L) \simeq[16$ $+10 L / 11] / 100$, providing the value of the ratio

$$
\frac{\alpha(L)}{\beta(L)} \simeq \frac{12-L / 15}{16+10 L / 11} .
$$

The delay $\tau_{1^{\prime} 1}$ indicates how much above or below the whale is situated to the hydrophone. It is positive if the whale swims its back toward the hydrophone and is negative if the whale swims its belly toward the hydrophone. It is equal to zero if the hydrophone is in the lateral plane of the whale.

\section{E. Pitch, yaw, and roll movements}

The off-axis angle indicates where the hydrophone is situated in relation to the whale. It is calculated from Eq. (5) using the measurement of the delay $\tau_{1 / 2}$. Assuming that the line $(D F)$ is close to the dorsorostral axis of the whale, the delay $\tau_{1 / 2}$, and, consequently, the off-axis angle are constant while the whale makes a roll movement. On the other hand, $\tau_{1^{\prime} 1}$ oscillates during such a movement around its mean value 0 and between the extrema given in Eq. (7). Variations of $\tau_{1^{\prime} 1}$ with $\tau_{1 / 2}$ being constant would then indicate a pure roll movement.

However, $\tau_{1 / 2}$ and $\delta$ are expected to vary during a pitch, a yaw, or a combination of pitch and yaw movements. Assuming $(J D) \perp(D F)$, the delay $\tau_{1^{\prime} 1}$ is constant during a yaw movement, whereas $\tau_{1 / 2}$ oscillates around its mean value, IPI/2. Variations of $\tau_{1 / 2}$ with $\tau_{1^{\prime} 1}$ being constant would then indicate a pure yaw movement.

Simultaneous variations of $\tau_{1 / 2}$ and $\tau_{1^{\prime} 1}$ indicate either a combination of roll and yaw movements, or a pitch movement, or a combination of roll, yaw, and pitch movements. Whereas synchronized variations of $\tau_{1 / 2}$ and $\tau_{1^{\prime} 1}$ would be the result of a pure pitch movement (Fig. 4). Such variations are similar (both increasing or both decreasing) if the hydrophone is located in front of and below the whale or behind and above the whale. Such variations are opposite (one increasing, one decreasing) if the hydrophone is located in front of and above the whale or behind and below the whale. By studying the variations of the delays $\tau_{1 / 2}$ and $\tau_{1^{\prime} 1}$ of a click sequence, one can qualitatively determine the nature of the whale's movements while it emits this click sequence.

\section{RESULTS}

\section{A. Multipulse structure}

A diagram illustrating the variations in the multipulse structure of the clicks emitted by a sperm whale during a single dive is provided in Fig. 5. Four sequences of 200 successive clicks are plotted, using the 2004 dataset presented in Laplanche et al. (2005). Only usual clicks (with ICI $>0.45 \mathrm{~s})$ are considered.

The $p 0$ pulse is clearly detected through the dive, given its low directionality (Zimmer et al., 2005b). The $p 1, p 1 / 2$, and $p 1^{\prime}$ pulses are also well detected. As predicted by the leaky bent-horn model, the $p 1 / 2$ and $p 1$ pulses are best detected if the hydrophone is in front of the whale ( $\delta$ $\in[0, \pi / 2] \Leftrightarrow \tau_{1 / 2} \in[\mathrm{IPI} / 2$, IPI $]$ ), the $p 1^{\prime}$ pulse is best detected if the hydrophone is behind the whale $(\delta \in[\pi / 2, \pi])$. The time of arrival of the $p 1$ and $p 1 / 2$ pulses fits with the model, as shown later using Eqs. (6) and (7), and clearly demonstrated by Zimmer et al. (2005a). The $p 1^{\prime}$ pulse is distinct with the $p 1$ pulse, as unequivocally illustrated in the first sequence of Fig. 5. As predicted by the bent-horn model, the $p 2^{\prime}$ pulse is noticeable only if $p 1^{\prime}$ is detected (e.g., in the first sequence of Fig. 5). The $p 3 / 2$ pulse is also detectable (e.g., in the third sequence of Fig. 5). Its travel time does not fit, however, with the model, as discussed later.

\section{B. Pulse delays and off-axis angle}

The travel time differences between the $\left\{p 0, p 1^{\prime}, p 2^{\prime}\right\}$ pulses are constant, as predicted by the model. The interpulse interval of the whale that clicks, plotted in Fig. 2 and Fig. 5, is $\mathrm{IPI}=5.8 \mathrm{~ms}$.

The application of Eqs. (6) and (7) requires the measurement of the whale's length $L$, the speed of sound $c_{s}$ in the spermaceti case, and the speed of sound $c_{w}$ in sea water at the recording location. The whale is of length $L=12.8 \mathrm{~m}$, using the empirical formula proposed by Rhinelander and Dawson (2004) with IPI=5.8 ms. The authors choose in the following a speed of sound $c_{s}=1370 \mathrm{~m} \mathrm{~s}^{-1}$ in the spermaceti 


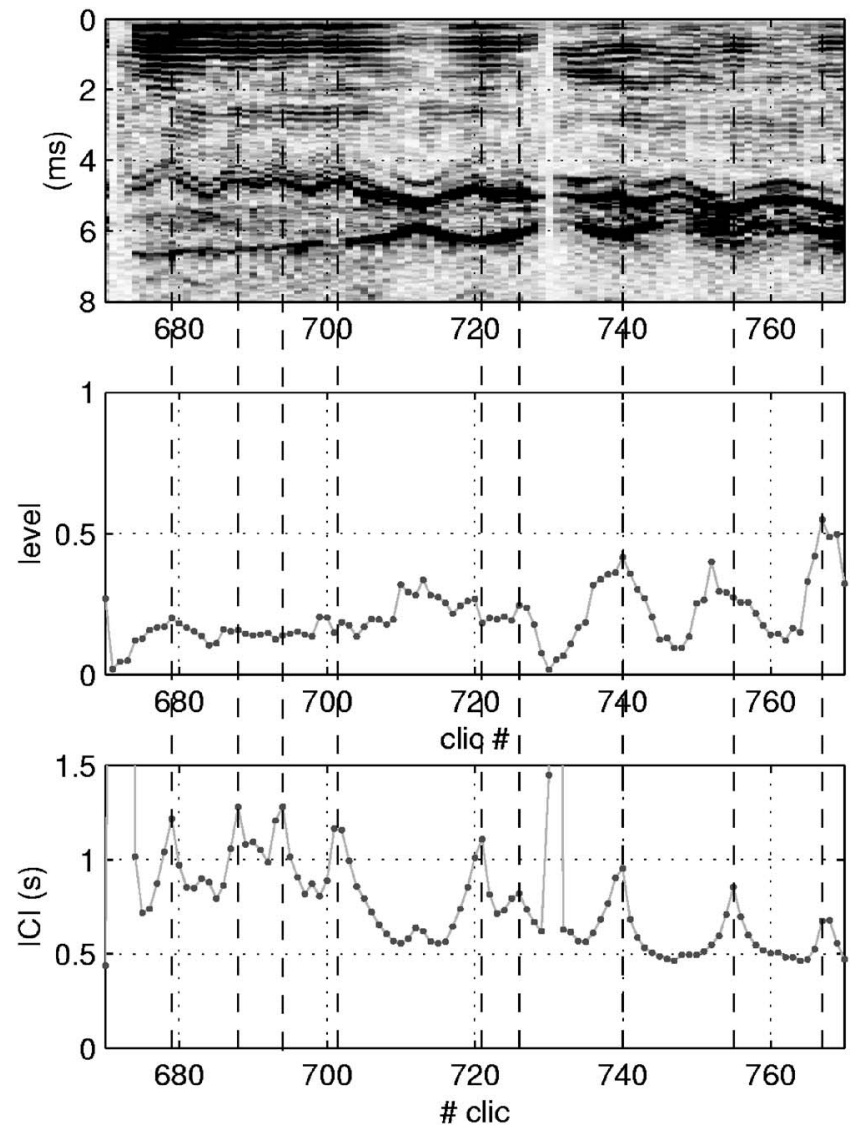

FIG. 6. The whale creates a fast yaw movement during the click sequence $\# 670 \rightarrow \# 730$ and a fast pitch movement during the click sequence \#730 $\rightarrow \# 770$. The ICI is in sync with the movement during the first sequence and in sync with the click apparent level during the second sequence.

organ, using the results presented by Flewellen and Morris (1978) for a temperature of $33{ }^{\circ} \mathrm{C}$ and a pressure of $1 \mathrm{~atm}$. Such a choice is discussed below. The speed of sound in seawater at the recording location was $c_{w} \simeq 1500 \mathrm{~m} \mathrm{~s}^{-1}$.

When applying Eq. (6), clicks are on axis and the hydrophone is in front of the whale $(\delta \in[0, \pi / 4])$ if 0.82 IPI $\leqslant \tau_{1 / 2} \leqslant \mathrm{IPI} \Leftrightarrow 4.8 \leqslant \tau_{1 / 2} \leqslant 5.8 \mathrm{~ms}$ and behind the whale $(\delta$ $\in[3 \pi / 4, \pi])$ if $0 \leqslant \tau_{1 / 2} \leqslant 0.18 \mathrm{IPI} \Leftrightarrow 0 \leqslant \tau_{1 / 2} \leqslant 1 \mathrm{~ms}$. Most of the clicks of the sequences of Fig. 5 are off axis $(\delta$ $\left.\in[\pi / 4,3 \pi / 4], 1 \leqslant \tau_{1 / 2} \leqslant 4.8 \mathrm{~ms}\right)$. Some clicks are almost on axis, for instance, click \#750, which is $\pi / 6$ off axis $\left(\tau_{1 / 2}\right.$ $=5.5 \mathrm{~ms}$ ), using (5) with $c_{\mathrm{sw}}=c_{s}$.

Using Eqs. (8) and (9), the delay of $p 1$ on $p 1^{\prime}$ should verify $\left|\tau_{1^{\prime} 1}\right| \leqslant 0.18 \mathrm{IPI} \simeq 1.05 \mathrm{~ms}$. Cranford (1999) suggested that the distance between the blow hole and the eye is a good estimate for the length of the spermaceti organ. Linear regressions of the results provided by Nishiwaki et al. (1963) then lead to a tip of snout to center eye distance close to $[15+26 L / 15] L / 100 \mathrm{~m}$, a tip of snout to blowhole distance close to $[3.5+4.5 \mathrm{~L} / 15] \mathrm{L} / 100 \mathrm{~m}$, hence a blowhole to center eye distance $\beta(L) L \simeq[11.5+21.5 L / 15] L / 100 \mathrm{~m}$. This leads to the estimate of the distance between the phonic lips and the location of point $J$ on the anterior surface of the junk $D J=\alpha(L) L=0.12 L=1.54 \mathrm{~m}$. Zimmer et al. (2005a) has found $D J=1.2 \mathrm{~m}$ for a sperm whale of length $L=12 \mathrm{~m}$. Given the similar lengths of the whales observed in both works, one should expect $\alpha(L)=0.10$ for the $12.8 \mathrm{~m}$ whale observed in the present work. This discrepancy comes from the fact that the height to length ratio of the whale's spermaceti organ is actually inferior to the height to length ratio of the whale's skull. This should be expected, in view of the relative importance of the maxillonasalis muscles in the constitution of the whale's snout (Clarke, 1978b). One should correct previous statements by writing $\alpha(L) / \beta(L)$ $=[\gamma(L) / \zeta(L)] / \eta$ with $\eta=1.2$. In that case, the delay of $p 1$ on $p 1^{\prime}$ should verify $\left|\tau_{1^{\prime} 1}\right| \leqslant 0.15 \mathrm{IPI} \simeq 0.9 \mathrm{~ms}$.

\section{Movements}

In the example presented in Fig. 5, the whale vertically dives to $400 \mathrm{~m}$ from click \#1 to click \#420 (Laplanche et al., 2005) (only clicks \#1 to \#200 of this descent are represented in Fig. 5). During this descent, both $\tau_{1 / 2}$ and $\tau_{1^{\prime} 1}$ delays oscillate, as the consequence of a physical movement of the whale. The whale barely rolls during the descent, the delay $\tau_{1^{\prime} 1}$ being always positive. The whale creates a slow pitch movement, resulting in the synchronous, slow variations of $\tau_{1 / 2}$ and $\tau_{1^{\prime} 1}$. Variations of $\tau_{1^{\prime} 1}$ during this pitch movement are in sync with the vertical movement described in Laplanche et al. (2005). Maxima of the delay $\tau_{1^{\prime} 1}$ (at $t$ $\in\{282,415,560\} \mathrm{s}$, clicks $\#\{125,245,380\})$ are detected during the inflections of the vertical movement of the whale [at $t \in\{280,400,570\} \mathrm{s}$, Fig. 5 of Laplanche et al. (2005)]. The slow pitch movement found using the present method and the vertical movement described in Laplanche et al. (2005) must represent the same slow, vertical, pitch movement. The whale also creates a repetitive and faster yaw movement (10-click period variations of $\tau_{1 / 2}$ with $\tau_{1^{\prime} 1}$ constant) during the descent.

Sperm whales also move actively when emitting usual clicks at depth. The variations of $\tau_{1 / 2}$ and $\tau_{1^{\prime} 1}$ being constant during the click sequence $\# 670 \rightarrow \# 730$ would result of a periodic yaw movement. Synchronous variations of $\tau_{1 / 2}$ and $\tau_{1^{\prime} 1}$ during the click sequence $\# 730 \rightarrow \# 770$ would result of a periodic pitch movement. The former movement is in sync with the click rhythm, as illustrated in Fig. 6. The authors suggest that the whale methodically scans the content of expanses of water during the descent and at depth, as discussed later.

\section{DISCUSSION}

\section{A. Multipulse structure}

The description of the multipulse structure proposed by the authors and Zimmer et al. (2005a) using the bent-horn model correctly predicts the existence, the time of arrival differences, and the relative levels of the pulses $\left\{p 0, p 1 / 2, p 1, p 1^{\prime}, p 2^{\prime}\right\}$. The model also predicts the existence of the $p 3 / 2$ pulse. The travel time difference of $p 3 / 2$ to $p 0$ is, however, shorter than expected. During the click sequence $\# 1000 \rightarrow \# 1025$, one can measure $\tau_{3 / 2} \simeq 8.1 \mathrm{~ms}$ (Fig. 5), though one should expect $\tau_{3 / 2}=\mathrm{IPI}+\tau_{1 / 2} \simeq 9.4 \mathrm{~ms}$. Whereas the delays $\tau_{1 / 2}$ and $\tau_{3 / 2}$ slightly change during this click sequence, the travel time difference $\tau_{3 / 2}-\tau_{1 / 2}$ of $p 3 / 2$ to $p 1 / 2$ remains constant. This suggest that the $p 3 / 2$ pulse is indeed the result of a multipath propagation inside the head of the whale and not the reflection by objects surrounding the 
whale. The travel time difference $\tau_{3 / 2}$ is, however, $1.3 \mathrm{~ms}$ lower than predicted by the model. According to the benthorn model, the $p 0$ pulse travels through the spermaceti case and reflects on the frontal sac ( $p 1 / 2$ pulse). The $p 1 / 2$ pulse leaks into the spermaceti junk (leading to the $p 1$ pulse) and case (leading to the $p 1^{\prime}$ pulse). This statement has lead the authors to speculate that the $p 1 / 2$ pulse might also leak in sea water. The distal reflection $p 1^{\prime}$, when clearly detected, is followed by an IPI-delayed pulse (the $p 2^{\prime}$ pulse). This has lead to the assumption that $p 1^{\prime}$ might follow an acoustic path inside the whale's snout similar to the one followed by $p 0$. The $p 1^{\prime}$ pulse would then reflect on the frontal sac, and, as $p 0 \mathrm{did}$, lead to a frontal reflection leaking in seawater: the $p 3 / 2$ pulse. The discrepancy underlined above regarding the value of the delay $\tau_{3 / 2}$ might be the consequence of the inexactitude of this model. The authors did not though manage to explain the reason of such discrepancy.

The $p 1 / 2$ pulse has been defined as the reflection of $p 0$ on the frontal sac. The $p 1 / 2$ pulse can, however, be detected using a hydrophone placed behind the whale (see, for instance, the fourth click sequence of Fig. 5). In such cases, the transmission of the $p 1 / 2$ pulse would reach and cross the frontal sac, before leaking in the sea water through the whale's thorax. The $p 1 / 2$ pulse has been described by Zimmer et al. (2005a) as arriving as scattered subpulses. Zimmer et al. (2005a) have suggested that this could be the result of multiple reflections on the frontal sac. The $p 1 / 2$ pulse is also, regarding the dataset used through this work, composed of subpulses when recorded in front of the whale (Fig. 6). It is, however, not time spread when detected from behind the whale. This would rather suggest that the $p 0$ pulse reaches the frontal sac at a single point. This does not forbid multipath propagation of $p 0$ within the spermaceti case, as it is expected to occur as a result of the heterogeneous temperature and chemical composition of the case (Flewellen and Morris, 1978). Leakage of the $p 0$ pulse through the frontal sac might reflect on the whale's lungs. Such reflections could result, using a hydrophone in front of the whale, of $p 1 / 2$ arriving as scattered subpulses. One should also expect, assuming multiple reflections on the frontal sac resulting from multipath propagation within the spermaceti case, $p 1 / 2$ to form a single broad spread pulse. Subpulses are, however, clearly identifiable (Fig. 6), bearing out the hypothesis describing $p 1 / 2$ as the result of multiple reflections on distinct surfaces. Such a hypothesis, however, does not take into account lung collapsing with pressure at depth, and does not explain why the sperm whale's frontal sac is large and parabolic. Such size and geometry might though indicate a double function of the frontal sac (e.g., an acoustic receptor, as suggested by Kozak et al.).

\section{B. Off-axis angle}

The off-axis angle is estimated using Eq. (6). The authors have assumed that (1) the speed of sound along the path $[D F]$ is constant through the dive and of known value $c_{s},(2)$ the path $[D F]$ is rectilinear and parallel to the click axis, (3) the speed $c_{\mathrm{sw}}$ along the path $[D K]$ (if $\delta \in[\pi / 2, \pi]$ ) or $[F K]$ (if $\delta \in[0, \pi / 2]$ ) is equal to the speed of sound in seawater $c_{w}$. The speed of sound in the core of the spermaceti case is expected to change through the dive, as a consequence of variations in temperature and pressure, $c_{s}(T, P)$ (Goold, 1996). Such variations could be the result of depthdependent variations of the thermodynamical properties of the sea water or the result of whale-controlled changes within the snout (thermoregulation or the use of the maxillonasalis muscles). The sperm whale is expected to control the temperature of its spermaceti case if it uses it to efficiently focus sounds (Flewellen and Morris, 1978), though it does not seem to have the required extensive vascularization system (Cranford, 1999). Anyone knows the precise variations of the temperature of the spermaceti case and junk through the dive. Gordon (1991) have considered $c_{s}$ $=1350 \mathrm{~m} \mathrm{~s}^{-1}$, Goold (1996) $c_{s}=1430 \mathrm{~m} \mathrm{~s}^{-1}$. The author's choice of $c_{s}=1370 \mathrm{~m} \mathrm{~s}^{-1}$ is here arbitrary, and has been chosen as the speed of sound in spermaceti at a temperature of $33{ }^{\circ} \mathrm{C}$ (Clarke, 1978a) and at the sea surface level.

In light of such spatial and temporal variations $c_{s}(x, y, z, t)$ within the spermaceti case, the path $[D F]$ is likely not rectilinear. The speed of sound $c_{s}$ used in Eq. (6) is not the mean speed of sound $c_{\mathrm{sc}}$ in the core of the spermaceti case along the curved path $[D F]$, but the speed required for an acoustic wave to travel rectilinearly between the points $D$ and $F$ with the travel time IPI/2. Assuming that the $p 0$ pulse reflects into $p 1 / 2$ at the center of the frontal sac, the line $(F D)$ is inclined relatively to the axis of the rostrum. The latter is, however, close to the axis of the junk. The speed of sound $c_{\mathrm{sw}}$ along the path $[D K]$ or $[F K]$ is actually a function of the off-axis angle, $c_{\mathrm{sw}}(\delta)$. The authors have assumed $c_{\mathrm{sw}}(\delta)=c_{s}$ if $|\delta-\pi / 2| \geqslant \pi / 4$ and $c_{\mathrm{sw}}(\delta)=c_{w}$ if $\pi / 4 \leqslant \delta$ $\leqslant 3 \pi / 4$. The knowledge of the spatial and temporal variations of the temperature within the spermaceti organ could resolve all the above approximations, leading to a precise measurement of (1) the speed of sound $c_{s}$, (2) the location of the reflection point $F,(3)$ the empirical law $c_{\text {sw }}(\delta)$.

The off-axis angle estimator proposed by the authors in Eq. (6) is then not accurate. Nevertheless, it is a beginning, and this work is worth being pursued. The applications of an off-axis angle estimator are various. One of them could allow to reconstruct the beam pattern of sperm whale clicks using a single hydrophone. The simultaneous measurements from different locations of the off-axis angle of a sperm whale could also be used to find the three-dimensional orientation (in the terrestrial reference frame) of this whale.

\section{Rotational movements}

Laplanche et al. (2005) have presented a model describing how sperm whales might move and echolocate while searching for prey. This model is partially correct. It is grounded on the hypothesis that sperm whales control click level and rhythm during echolocation. Such a hypothesis has been made in light of the apparent synchronization of click level and ICI (for instance, in Fig. 7 of this reference). Nevertheless, such synchronization could result when the whale synchronizes its click rhythm to an oscillatory movement, and when the observer's position is less than ideal. Laplanche et al. (2005) rejected such a hypothesis, thinking 

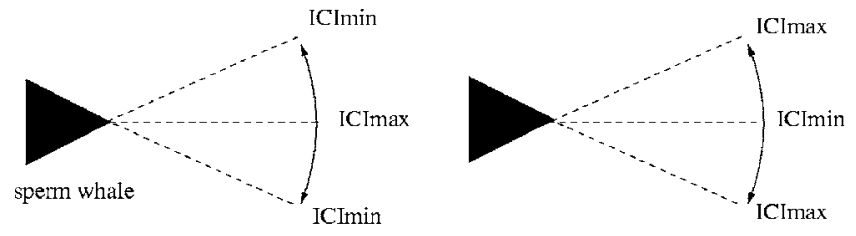

(c)
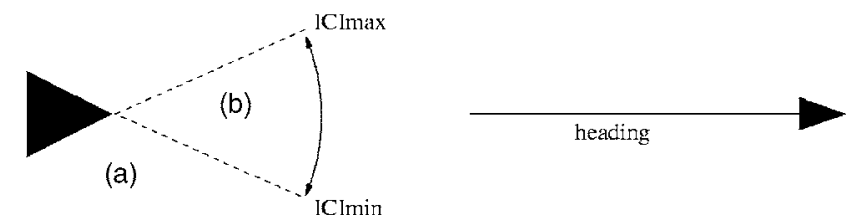

FIG. 7. Three models describing the fast, periodic movement of the whale in sync with its ICI. Only the third model could lead to the synchronization $\mathrm{ICI}_{\max } \Leftrightarrow \delta_{\max }$ using a hydrophone located either in front of or behind the whale [it is located in (a)]. The click apparent level and the ICI are in sync $\left(\mathrm{ICI}_{\max } \Leftrightarrow \mathrm{AL}_{\max }\right)$ if the hydrophone is located in (c). There is no apparent synchronization between the ICI, the off-axis angle, and the click AL if the hydrophone is in sector (b).

that an ICI-movement synchronization would lead to an ICIlevel synchronization only for on-axis recordings. Actually, the lateral asymmetry in the acoustic behavior of sperm whales while searching for prey described later could explain why click level and rhythm can so often be in apparent sync.

As illustrated regarding the click sequence \#670 $\rightarrow \# 730$ (Fig. 6), the sperm whale clicking activity can be in sync with the fast, periodic movement it creates (in this case a yaw movement). In this example, the hydrophone is in front of the whale $(\delta \leqslant \pi / 2)$ and the maxima of the ICI correspond to the maxima of the off-axis angle. A similar relationship (i.e., $\mathrm{ICI}_{\max } \Leftrightarrow \delta_{\max }$ ) can be observed using a hydrophone behind the whale $(\delta \geqslant \pi / 2)$.

The authors think of three models describing the fast, periodic, rotational movement of the whale in sync with its ICI (Fig. 7). Such a rotational movement is assumed to be planar in the following. The first model stipulates that the whale echolocates at slow rate $\left(\mathrm{ICI}_{\max }\right)$ in front of it and faster $\left(\mathrm{ICI}_{\min }\right)$ on its sides. The second model stipulates that the whale echolocates at high rate $\left(\mathrm{ICI}_{\min }\right)$ in front of it and slower $\left(\mathrm{ICI}_{\max }\right)$ on its sides. The third model stipulates that the whale echolocates at slow rate $\left(\mathrm{ICI}_{\max }\right)$ on one side and faster $\left(\mathrm{ICI}_{\min }\right)$ on the other.

One can show that during a periodic, planar, rotational movement, the off-axis angle reaches its extreme, either when the hydrophone is in the plane containing the whale dorsorostral axis and the rotational axis or during the inflections of the movement. Whereas the synchronization of the ICI and click level may appear approximate (e.g., during the click sequence $\# 730 \rightarrow \# 770$ of Fig. 6), the synchronization of the ICI and the off-axis angle is unequivocal (e.g., during the click sequence $\# 670 \rightarrow \# 730$ ). The authors, whereas they should have not so easily rejected the hypothesis that the ICI-apparent level synchronization being the result of the hydrophone being in a less than ideal location [Laplanche et al. (2005)], here they reject the hypothesis that the ICI- $\delta$ synchronization mentioned above is apparent only. They assume in the following that the extreme of the off-axis angle (as those illustrated in Fig. 6) are the consequence of inflections in the rotational movement of the whale.
One can then show that only the third model could lead to an $\mathrm{ICI}_{\max } \Leftrightarrow \delta_{\max }$ synchronization using a hydrophone located either in front of or behind the whale. Such an important result, an asymmetry in the use of the sperm whale's biosonar, need to be borne out by analyzing more data before being considered as representative of the sperm whale acoustic behavior. It could be, for instance, the result of the asymmetry in the anatomy of the sperm whale sould generator, or part of a metabolic saving foraging strategy (Laplanche, 2005).

Such an asymmetry in the sperm whale acoustic behavior also explains the often observed synchronization of the ICI and apparent click level (AL). As illustrated in Fig. 7, the relationship $\mathrm{ICI}_{\max } \Leftrightarrow \mathrm{AL}_{\max }$ is detectable using a hydrophone located in a large portion of space [on side (C)]. The ICI- $\delta$-AL appear to be decorrelated when using a hydrophone in front of the whale [in sector (B)].

The technique presented in Laplanche et al. (2005) has been used to measure the vertical movements of diving sperm whales. It does not detect horizontal movements, hence the inexactitude of the model previously presented by the authors. However, the existence of horizontal movements does not alter the measurement of vertical movements, and the technique used by Laplanche et al. (2005) remains valid. The description of the vertical movements of sperm whales while descending and foraging at depth is then to be supplemented with the description of their horizontal movements.

The sperm whale, regarding the dive chosen as an example, makes a slow vertical pitch movement and a faster yaw movement with no rolling during the descent. Data presented by Zimmer et al. (2005a) would rather suggest that sperm whales create a fast pitch movement during the descent as a result of fluking. The fast movement, has, however, not been detected by Laplanche et al. (2005), suggesting that it is horizontal. The authors' present results suggest that, in the example illustrating this work, the whale would analyze the content of a horizontal water expanse during this descent, by making a slow, vertical, pitch movement and create a faster, horizontal, yaw movement.

At depth, regarding the sequence \#670 $\rightarrow \# 730$ (Fig. 6), the whale makes a slow vertical pitch movement, barely rolls, and creates a faster yaw movement. The latter movement would be horizontal, for reasons similar to those mentioned above, though this statement is highly speculative, because Laplanche et al. (2005) have used a different dataset to show that sperm whales would not create fast, vertical movements during the searching phases. The whale would, in this case, analyze the content of a vertical water expanse. Concerning the sequence $\# 730 \rightarrow \# 770$ (Fig. 6), the fast movement would be a pitch movement.

The authors have not analyzed enough data to conclude on how sperm whales precisely move when searching for prey. The method itself is qualitative, as a result of making 2 measurements- $\tau_{1 / 2}$ and $\tau_{1^{\prime} 1}$-when trying to estimate three angles-pitch, yaw, and roll. The authors could not use it to precisely determine the geometry of the water masses analyzed by sperm whales when foraging. Nevertheless, the method presented is promising, and the first results demon- 
strate that sperm whales actively search for their prey, by creating a fast, periodic movement in sync with their acoustic activity.

\section{SUMMARY AND CONCLUSIONS}

The traditional view of the sperm whale click multipulse structure describes a click as a regularly spaced series of pulses $\{p 0, p 1, p 2, p 3, \ldots\}$. Nevertheless, additional pulses are often detected within this structure. Their presence has been explained using the standard bent-horn model of the sperm whale sound generator. Frontal reflections $\{p 1 / 2, p 3 / 2, p 5 / 2, \ldots\}$ and distal reflections $\left\{p 1^{\prime}, p 2^{\prime}\right.$, $\left.p 3^{\prime}, \ldots\right\}$ would also leak from the sperm whale snout, leading to a more complex sperm whale click multipulse structure when recorded by a farfield, off-axis hydrophone.

The delays $\tau_{1 / 2}$ (of $p 1 / 2$ on $\left.p 0\right)$ and $\tau_{1^{\prime} 1}$ (of $p 1$ on $p 1^{\prime}$ ) are variable and depend on the whale's orientation to the hydrophone. The off-axis angle of the whale can be calculated using the measurements of the standard IPI and the delay $\tau_{1 / 2}$. The nature of the movements of the whale (if it is pitch, yaw, or roll) can be measured by analyzing the variations of the delays $\tau_{1 / 2}$ and $\tau_{1^{\prime} 1}$. Variations of $\tau_{1 / 2}$ alone (with $\tau_{1^{\prime} 1}$ constant) indicate pure yaw movements, variations of $\tau_{1^{\prime} 1}$ alone (with $\tau_{1 / 2}$ constant) indicate pure roll movements, and synchronous variations of $\tau_{1 / 2}$ and $\tau_{1^{\prime} 1}$ indicate pure pitch movements. The analysis of the waveform of usual click sequences then leads to some insight on the sperm whale movements when foraging.

Sperm whales would barely roll when descending and when searching for prey at depth. In the example illustrating this work, the authors have shown that the whale made a slow vertical pitch movement and at the same time created a faster, periodic yaw movement when descending. When searching for prey at depth, the sperm whale made a slow pitch movement and created a faster pitch or yaw movement in sync with the clicking activity. The authors have suggested that sperm whales would, at depth, make an asymmetric scan of the surrounding water. The method presented is easily implemented, is repeatable, and could lead to new insights on the foraging behavior of sperm whales or other toothed whales using a very simple apparatus consisting of a single hydrophone.

\section{ACKNOWLEDGMENTS}

Thanks to L. Conteville (Université Paris XII, France) for helping in analyzing the data. Recordings could not have been made without the help of the Association Breach (http:// www.breach-asso.org, France), which provided boats, skippers, and ecovolunteers. Thanks to the Association Keto $\Sigma$ (France) for undertaking the airborne survey. Many thanks to $\mathrm{S}$. Wiener for reviewing an earlier version of the manuscript and to L. Adam, M. Adam, and C. Bédard for refining the English. The authors also thank M. Macé for his help on the bibliography and W. Zimmer for his useful and encouraging comments on the accepted version of the manuscript.

Backus, R., and Schevill, W. (1966). "Physeter Clicks," in Whales, Dolphins and Porpoises, edited by K. Norris (University of California Press, Berkeley), pp. 510-527.
Bahl, R., Ura, T., and Fukuchi, T. (2002). "Towards identification of sperm whales from their vocalizations," Seisan-Kenkyu bimonthly J. of IIS, University of Tokyo, Vol. 54-6, pp. 51-55.

Clarke, M. (1978a). "Buoyancy control as a function of the spermaceti organ in the sperm whale," J. Mar. Biol. Assoc. U.K. 58, 27-71.

Clarke, M. (1978b). "Structure and proportions of the spermaceti organ in the sperm whale," J. Mar. Biol. Assoc. U.K. 58, 1-17.

Cranford, T. (1999). "The sperm whale's nose: Sexual selection on a grand scale?," Marine Mammal Sci. 15, 1133-1157.

Flewellen, C., and Morris, R. (1978). "Sound velocity measurements on samples from the spermaceti organ of the sperm whale (Physeter catodon)," Deep-Sea Res. 25, 269-277.

Goold, J. (1996). "Signal processing techniques for acoustic measurement of sperm whale body lengths," J. Acoust. Soc. Am. 100, 3431-3441.

Gordon, J. (1991). "Evaluation of a method for determining the length of sperm whales (Physeter catodon) from their vocalizations," J. Zool. 224, 301-314.

Jaquet, N., Dawson, S., and Douglas, L. (2001). "Vocal behavior of male sperm whales: Why do they click?" J. Acoust. Soc. Am. 109, 2254-2259.

Kawakami, T. (1980). "A review of sperm whale food," Sci. Rep. Whales Res. Inst. (Tokyo) 32, 199-218.

Laplanche, C. (2005). "Etude par acoustique passive du comportement de chasse du grand cachalot," Ph.D. thesis, Université Paris 12, Créteil.

Laplanche, C., Adam, O., Lopatka, M., and Motsch, J. (2005). "Male sperm whale acoustic behavior observed from multipaths at a single hydrophone," J. Acoust. Soc. Am. 118, 2677-2687.

Lockyer, C. (1981). "Estimates of growth and energy budget for the sperm whale," Physeter catodon, in Mammals in the Seas, vol. 3, FAO series No. $5,489-504$

Madsen, P., Johnson, M., Aguilar de Soto, N., Zimmer, W., and Tyack, P. (2005). "Biosonar performance of foraging beaked whales (Mesoplodon densirostris)," J. Exp. Biol. 208, 181-194.

Madsen, P., Payne, R., Kristiansen, N., Wahlberg, M., Kerr, I., and Møhl, B. (2002). "Sperm whale sound production studied with ultrasound time/ depth-recording tags," J. Exp. Biol. 205, 1899-1906.

Miller, P., Johnson, M., and Tyack, P. (2004). "Sperm whale behavior indicates the use of echolocation click buzzes 'creaks' in prey capture," Proc. R. Soc. London, Ser. B 271, 2239-2247.

Møhl, B., Wahlberg, M., Madsen, P., Heerfordt, A., and Lund, A. (2003). "The monopulsed nature of sperm whale clicks," J. Acoust. Soc. Am. 114, 1143-1154.

Møhl, B., Wahlberg, M., Madsen, P., Miller, L., and Surlykke, A. (2000). "Sperm whale clicks: Directionality and source level revisited," J. Acoust. Soc. Am. 107, 638-648.

Mullins, J., Whitehead, H., and Weilgart, L. (1988). "Behavior and vocalizations of two single sperm whales, Physeter macrocephalus, off nova scotia," Can. J. Fish. Aquat. Sci. 45, 1736-1743.

Nishiwaki, N., Ohsumi, S., and Maeda, Y. (1963). "Change of form in the sperm whale accompanied with growth," Sci. Rep. Whales Res. Inst. (Tokyo) 17, 1-14.

Norris, K., and Harvey, G. (1972). "A theory for the function of the spermaceti organ of the sperm whale (Physeter catodon L.)," in Animal Orientation and Navigation, edited by S. Galler, K. Schmidt-Koenig, G. Jacobs, and R. Belleville (NASA, Washington, D.C.), pp. 397-417.

Rhinelander, M., and Dawson, S. (2004). "Measuring sperm whales from their clicks: Stability of interpulse intervals and validation that they indicate whale length," J. Acoust. Soc. Am. 115, 1826-1831.

Urick, R. (1983). Principles of Underwater Sound (McGraw-Hill, New York), 3rd ed..

Watkins, W., Dahar, M., DiMarzio, N., Samuels, A., Wartzok, D., Fristrup, K., Gannon, D., Howey, P, and Maiefski, R. (1999). "Sperm whale surface activity from tracking by radio and satellite tags," Marine Mammal Sci. 15, 1158-1180.

Whitehead, H. (2003). Sperm Whales: Social Evolution in the Ocean (The University of Chicago Press, Chicago).

Whitehead, H., and Weilgart, L. (1990). "Click rates from sperm whales," J. Acoust. Soc. Am. 87, 1798-1806.

Zimmer, W., Madsen, P., Teloni, V., Johnson, M., and Tyack, P. (2005a). "Off-axis effects on the multipulse structure of sperm whale usual clicks with implications for sound production," J. Acoust. Soc. Am. 118, 33373345.

Zimmer, W., Tyack, P., Johnson, M., and Madsen, P. (2005b). "Threedimensional beam pattern of regular sperm whale clicks confirms benthorn hypothesis," J. Acoust. Soc. Am. 117, 1473-1485. 